\title{
Las comunidades de práctica en las organizaciones
}

\section{Communities of practice in organizations}

\author{
Manuel Alfonso Garzón Castrillón \\ Fundación para la Investigación y el Desarrollo Educativo Empresarial FIDEE \\ Barranquilla, Colombia.
}

\section{Autores de Correspondencia}

manuelalfonsogarzon@fidee.org

Recibido: 20-10-2019

Aceptado: 24-01-2020

\section{Resumen}

En este artículo de revisión, se abordan en primer lugar, las comunidades, los elementos que las conforman, sus características, tipos de comunidades, Comunidades de Práctica (CoP) y sus antecedentes. Posteriormente, se hace un acercamiento al concepto de comunidades de práctica, se propone la definición y un modelo que orientará el documento resultado de la revisión bibliográfica. Seguidamente, se realizan aproximaciones a las orientaciones para el diseño $y$, sobre todo, la implementación y su ciclo de vida, de la misma forma se identifican factores que condicionan el éxito, los componentes, el rol del moderador-dinamizador. Finalmente, se resumen las conclusiones pertinentes.

Palabras clave: Aprendizaje organizacional, entornos de trabajo, gestión del conocimiento. Clasificación JEL: D23

\section{Abstract}

In this review paper, we first address the communities, the elements that constitute them, their features, the types of communities, communities of practice (CoP), and their backgrounds. Subsequently, is followed by an approach to the concept of CoPs, followed by a definition and a model proposed to guide the document to the outcome according to the literature review. Next, the guidelines for design, implementation, and lifecycle are drawn and the factors that condition success, the components, and the role of the moderator-driving individual are identified. Finally, pertinent conclusions are outlined.

Keywords: Organizational learning, working environments, knowledge management. JEL Classification: D23

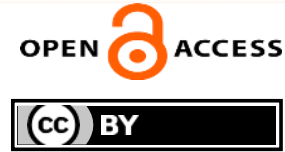

Copyright (C) 2020

Desarrollo Gerencial
Como citar este artículo (Apa):

Garzón, M. (2020). Las comunidades de práctica en las organizaciones. Desarrollo Gerencial, 12(1), 1-22. DOI:

https://doi.org/10.17081/dege...3683 


\section{Introducción}

La interacción social y las relaciones que se establecen con los compañeros de trabajo son las que permiten crear habilidades y dinamizar el conocimiento; estas a su vez se ven reflejadas en las comunidades de práctica, las cuales son reconocidas como las impulsoras del aprendizaje en las organizaciones, logrando construir una historia común desde las relaciones interpersonales. Además, permiten formular sus propias de formas de comunicación, instituir modos de resolver los problemas que se presentan tanto en lo personal como en lo laboral, generar compromisos de equipo y formas de dar solución a los problemas que se presenten en el día a día desarrollando una visión prospectiva alineada con la de la organización.

De acuerdo con lo anterior, este artículo de revisión aborda en primer lugar, las comunidades, los elementos que las conforman, sus características, los tipos de comunidades, las comunidades de práctica (CoP) y sus antecedentes, posterior a ello, se presenta un acercamiento al concepto de comunidades de práctica, luego se propone la definición y un modelo que orienta al documento resultado de la revisión bibliográfica. Seguidamente, se realizan unas aproximaciones a las orientaciones para el diseño y sobre todo, la implementación y su ciclo de vida. De la misma forma, se identifican los factores que condicionan el éxito, los componentes y el rol del moderador-dinamizador. Por último, se resumen las conclusiones correspondientes al estudio.

\section{Fundamentación teórica}

\section{Comunidades.}

Dentro de la literatura, en lo relacionado con el termino comunidad, Hilley (1955) plantea que existen un gran número de definiciones, pero que no se ha logrado determinar una definición como tal, sin embargo, este autor señala que se conciben como grupos de personas que tienen un vínculo común y que se caracterizan por estar en un entorno cercano. De esta aproximación se destaca que además deben estar agrupados alrededor de un interés común, lo que coincide con las comunidades de aprendizaje (CoP).

El origen del término comunidad se ubica en la locución latina communis que significa común (Corominas, 1987) y se refiere a grupos de personas identificadas por algo que es recíproco, que también está relacionada con co-munio, la cual es entendida como entre todos.

En este orden de ideas, la expresión comunidad, difiere con el termino de asociación, ya que para Tönnies (1895) son formas disímiles de agrupación, donde en la primera (comunidad), se refiere a grupos 


\section{Manuel Garzón Castrillón}

que están relacionados con entornos rurales como las congregaciones familiares y se caracterizan, por la cercanía, la afinidad en la forma de vida y las usanzas parecidas, mientras que en la segunda( asociación), se caracteriza por tener una distancia física con distintas experiencias, pero que tienen un trato transitorio que subsiste mientras se mantienen intereses comunes y compartidos.

Por otra parte, desde la perspectiva de Wilbur (1997), comunidad es un grupo de personas que se congregan y comparten de manera frecuente particularidades, fines, características, identificaciones, entre otros, en espacios que comparte en común. En esta definición se destacan la ubicación geográfica de proximidad espacial.

Como se puede inferir, el concepto de comunidad presenta muchas formas de comprensión, pero que todas confluyen en que, para que éstas existan se requiere que haya un sentimiento de pertenencia a la comunidad, por parte de los miembros que la conforman, como fundamento psicológico, que incluye la identidad del miembro y de la comunidad, sintiendo el compromiso de formar parte de ella y compartiendo un espacio común (Pons, et ál. 1996).

Así de esta manera, según McMillan y Chavis (1986) son cinco elementos que le dan significado a una comunidad, las cuales son: sentido de pertenencia y seguridad; influjo en las dos direcciones del miembro a la comunidad y de la comunidad para con él; participación; unificación de sus necesidades y las de los miembros y la satisfacción de estas necesidades; y vínculo emocional compartido interactivo.

De acuerdo con lo anterior, la Tabla 1, presenta los cuatro requisitos para conformar una comunidad:

\section{Tabla 1}

\section{Requisitos para conformar una comunidad}

\begin{tabular}{|c|c|c|}
\hline & Requisitos & Definición \\
\hline 1 & La ubicación geográfica & $\begin{array}{l}\text { El lugar concreto y social en el que se desarrollan las interrelaciones de los } \\
\text { integrantes de la comunidad }\end{array}$ \\
\hline 2 & Aspectos demográficos & $\begin{array}{l}\text { Características que posibilite clasificación, como edad, sexo, situación económica, } \\
\text { etc., necesario para estructurar y planificar la comunidad }\end{array}$ \\
\hline 3 & La demanda & $\begin{array}{l}\text { Por existencia de un problema, es demanda explícita, o por observación del } \\
\text { problema con una magnitud mayor al de la demanda que está latente, por } \\
\text { demanda implícita }\end{array}$ \\
\hline 4 & Los recursos & $\begin{array}{l}\text { Que posee la comunidad, económicos, de apoyo institucional, cultural o social, } \\
\text { como elemento definitivo para el impulso de la comunidad }\end{array}$ \\
\hline
\end{tabular}

Fuente: Elaboración propia (2018) con base en McMillan y Chavis (1986).

Asimismo, Garber (2004) señala que una comunidad es un grupo de personas que se aglomeran en un lugar o en algunos lugares con una razón que los une y cuyo fin es intervenir en algo que tiene en común, pero que definitivamente es superior a tener objetivos comunes, estos encuentros espontáneos, forman parte de una red de relaciones sociales y acciones compartidas. 
De esta definición propuesta por Garber se desprenden unas características que debe cumplir una comunidad que quiera destacarse (Tabla 2).

\section{Tabla 2}

\section{Componentes de una comunidad}

\begin{tabular}{|c|c|c|}
\hline & Componentes de una comunidad & Definición \\
\hline 1 & El núcleo que le da sentido. & $\begin{array}{l}\text { Con un propósito compartido que le otorga identidad y establece los roles de } \\
\text { los individuos participantes. }\end{array}$ \\
\hline 2 & Apropiación & $\begin{array}{l}\text { Pertenecer a una comunidad produce compromiso en sus miembros hacía los } \\
\text { fines de esta. }\end{array}$ \\
\hline 3 & $\begin{array}{l}\text { Más que un entramado o red de } \\
\text { relaciones }\end{array}$ & $\begin{array}{l}\text { Se logra una identidad que comparten para lograr un objetivo común, mediante } \\
\text { red de relaciones, dando valor a lo que obtienen. }\end{array}$ \\
\hline 4 & $\begin{array}{l}\text { Uso de diferentes medios para } \\
\text { comunicarse. }\end{array}$ & Se usan canales virtuales en tiempo real o en cualquier momento. \\
\hline 5 & Relaciones orientadas a resultados & $\begin{array}{l}\text { Se logra interacción y diálogo consolidan la vida de la comunidad, creando } \\
\text { vínculos que generan resultados para ésta y sus miembros. }\end{array}$ \\
\hline 6 & $\begin{array}{l}\text { Propósito común y actividades } \\
\text { compartidas. }\end{array}$ & $\begin{array}{l}\text { Definen procesos participativos orientados a la consecución del propósito } \\
\text { común, que caracterizan las actividades que se realizan en la comunidad } \\
\text { incluyendo normas de comportamiento. }\end{array}$ \\
\hline
\end{tabular}

Fuente: Elaboración propia (2018) con base en Garber (2004).

A su vez, los elementos clave de una comunidad relacionados en la Tabla 2, se complementan con los propuestos por Poole (2002) (Tabla 3).

\section{Tabla 3}

Tipologías de una comunidad

\begin{tabular}{|c|c|c|}
\hline & Tipologías & Definición \\
\hline 1 & Experiencias compartidas & $\begin{array}{l}\text { Orientadas a lograr sentido de pertenencia al compartir actividades y } \\
\text { vivir una experiencia común. }\end{array}$ \\
\hline 2 & Corresponsabilidad & Responsabilidad compartida de la trayectoria de la comunidad. \\
\hline 3 & Cultura compartida & $\begin{array}{l}\text { Tienes valores y principios que comparten, que se manifiestan en interés } \\
\text { comunes con sentido de pertenencia. }\end{array}$ \\
\hline 4 & Tiempo social & $\begin{array}{l}\text { Comparten tiempos de recreación que propician estrechar lazos, } \\
\text { fomentan la participación general. }\end{array}$ \\
\hline 5 & Costumbre de bienvenida y despedida & $\begin{array}{l}\text { Fomenta sentido de pertenencia, genera sensación de bienestar y } \\
\text { colaboración, permite acciones reciprocas. }\end{array}$ \\
\hline 6 & Capacidad de asumir compromiso y confiar & $\begin{array}{l}\text { Descubren el valor que representa su participación, refuerza la } \\
\text { responsabilidad con la comunidad, valoran sus aportes. }\end{array}$ \\
\hline 7 & Participación & $\begin{array}{l}\text { La intervención permanente genera integración, en sentido contrario } \\
\text { muestra que no se ha integrado. }\end{array}$ \\
\hline
\end{tabular}

Fuente: Elaboración propia (2018) con base en Poole (2002). 
Igualmente, autores como Coll et ál. (2007) afirman que las comunidades en general se conforman por personas con intereses compartidos y rasgos particulares, que se comportan de forma activa al involucrarse e intervenir conjuntamente en las acciones que se realizan en ella, con objetivos concreto frecuentemente, aunque no necesariamente, algunos forman parte porque tiene en común la edad, el oficio o la carrera profesional, la ubicación geográfica, el idioma y en general aspectos culturales.

Hamman (2001), señala también que, las comunidades están orientadas desde un enfoque social porque forman parte de ella, estas les genera un sentido de pertenencia a las personas que lo conforman, ya que comparten lugares y épocas en concretas.

\section{Tipologías de comunidades.}

Con base en los supuestos de Wellman y Gulia (1999) y dependiendo del propósito que promueven se identificaron cuatro tipos de comunidades, las cuales se muestran en la Tabla 4.

\section{Tabla 4.}

\section{Tipología de comunidades}

\begin{tabular}{|c|c|c|}
\hline \multicolumn{3}{|c|}{ Definición } \\
\hline 1 & Comunidades temáticas & $\begin{array}{l}\text { Se utilizan para la obtención y permuta de información sobre temas } \\
\text { específicos. }\end{array}$ \\
\hline 2 & Comunidades espaciales & $\begin{array}{l}\text { Se conforman por personas que se encuentra en lugares geográficos cercanos, } \\
\text { con intereses comunes, lo cual genera sentido de pertenencia }\end{array}$ \\
\hline 3 & Comunidades de aprendizaje (CdA) & $\begin{array}{l}\text { Se organizan por personas que tienen interés en intercambiar y lecciones } \\
\text { aprendidas para construir conocimiento colectivo, que genere beneficios para } \\
\text { sus miembros, aprendizaje permanente, motivación en conjunto. }\end{array}$ \\
\hline 4 & Comunidades de práctica (CoP) & $\begin{array}{l}\text { Se conforman por individuos con propensión a compartir buenas prácticas, que } \\
\text { resuelvan problemas en otras áreas y se reúnen periódicamente. }\end{array}$ \\
\hline
\end{tabular}

Fuente: Elaboración propia (2018) con base en Wellman y Gulia, (1999) y Wenger et ál., (2002).

\section{Comunidades de práctica.}

\section{Orígenes.}

El concepto de comunidades de práctica (CoP) tiene sus orígenes en los autores Wenger (2001), quienes hacen alusión a las características de este tipo de comunidades basadas en un proceso de aprendizaje continuo, en las cuales se concibe la apropiación de conocimientos como un proceso social, resaltando que se requiere formar parte de esta comunidad para adquirir conocimientos de construcción colectiva a diferencia del aprendizaje individual.

En este proceso de revisión bibliográfica se encontraron que otros autores como Brown y Guguid (1991), a quienes se les confiere la paternidad del término comunidades de práctica desarrollaron un 


\section{Manuel Garzón Castrillón}

estudio basado en las experiencias realizadas en la empresa Xerox y en la cual implementaron comunidades de práctica en las actividades diarias.

Asimismo, Wenger (1998) establece unas características particulares para las comunidades de práctica: compromiso bilateral; empresa vinculada y conocimiento compartido.

Desde la perspectiva de Lesser y Prusak (1999) se establece que las CoP son importantes para impulsar el aprendizaje en las organizaciones, en razón a que impulsan el capital social y construyen comparte y utilizan el conocimiento generado.

Para Jubert (1999), las CoP son "un grupo de personas que se reúnen de manera informal para compartir su experiencia y pasión por una empresa en común" (p. 24). Además, en este tipo de comunidades se resalta el conocimiento de las personas que forman parte de ellas, y se logra dinamizando en el lugar de trabajo los procesos de aprendizaje basados en la interacción social (Choo, 1999).

Por otra parte, García (2000), caracteriza a las CoP como un grupo social en el cual cada miembro participa de forma diferente, pero es unido al compartir el desarrollo de una actividad. Wenger y Snyder (2000), resalta que esta unión de los miembros de estas comunidades se da por una práctica común repetida y continúa lo que genera un aprendizaje.

En la definición que proponen Wenger et ál., (2002), estas son concebidas como "un grupo de personas que comparten una preocupación, un conjunto de problemas o un interés común acerca de un tema, y que profundizan su conocimiento y pericia en esta área a través de una interacción continuada" (p. 4), lo cual coincide con la perspectiva propuesta por Pickett (2002) en lo relativo a la identidad común de sus integrantes y los fines que los une, lo cual permite una relación de camaradería que les lleva a compartir y hacer parte de ella.

Para Vásquez citado en Sanz (2005), las CoP son "un grupo cuyos miembros coincide para compartir y aprender de sus interés comunes" (p.28), que en coincidencia con Wenger et ál. (2002), comparten inquietudes, dificultades y experticia en su área de trabajo mediante una continua interrelación, que se complementa con la propuesta de Cornejo y Medina citados en Gallego y Valdivia (2013), al establecer que deben ser personas especializadas en una misma área de trabajo y que se unen para compartir conocimientos y actividades laborales que desarrollan, mediante las reuniones habituales, sin importar si son formales o informales.

Asimismo, desde la propuesta de Medellín (2003), las CoP las personas que las conforman coinciden en aspectos como: intereses o áreas particulares, conocimientos tácitos, pues están en la cabeza de los 


\section{Manuel Garzón Castrillón}

individuos, que no tiene una estructura formal y no están estandarizados, pero que al utilizarlos en comunidad impulsan cambios importantes en los ecosistemas internos de las organizaciones.

Zeller citado en Gallego y Valdivia (2013), señala también que las CoP comparten experiencias, intereses que los miembros tienen sobre una temática, en forma continua lo cual permite profundizar en su aprendizaje, sus miembros resuelven problemas a través de discusiones e intercambio de información y conocimientos, desarrollando herramientas y marcos de referencia que aumentan el conocimiento que la comunidad tiene.

Las CoPs para Wenger (1998) permiten que los individuos que las conforman compartan ideas, busquen y logren soluciones, y generen innovación, para lo cual se requiere de la unión de esfuerzos de sus miembros, aprendiendo de todas las formas presencial y virtual, que, amalgamados por el propósito común de compartir experiencias, conocimientos y buenas prácticas sobre un área de trabajo, un tema 0 un área del conocimiento en particular.

La propuesta de Chen, Hsin y Seng (2007) aporta a la conceptualización de las CoPs, que forman parte de la organización informal, lo cual incentiva el intercambio de conocimientos, destrezas y practicas incentivadas desde la informalidad, coincidiendo con Wenger y Synder (2000) y Lesser y Stork (2001), quienes además le adicionan autoorganizadas, basadas en programación de temas, que eligen de sus propios líderes, y flexibles. Estos aportes se complementa con el trabajo realizado por Rodríguez (2008), quien las concibe como organizaciones que permanecen durante un período de tiempo, que además comparten unas prácticas entre los que la conforman y generan más resultados que las comunidades virtuales esporádicas.

Teniendo como base las diferentes definiciones anteriormente analizadas, y al encontrar que no hay acuerdos en éstas, se propone una definición de Comunidades de Prácticas (COP), la cual orienta el propósito de este artículo. Así, las CoP se definen como las comunidades que son promovidas por personas e impulsadas por directivos de las organizaciones, las cuales se auto-organizan y se conforman de manera informal. Estas están ligadas a actividades comunes que surge y subsisten en condiciones difíciles, funcionan incluso en organizaciones jerarquizadas que están orientadas a la realización de acciones comunes, compartiendo buenas prácticas que permiten el aprendizaje (aprender haciendo), logrando la competencia de sus miembros, generando innovación e identidad que las diferencia por su sentido de comunidad.

De la definición anteriormente propuesta surge el siguiente modelo de comunidades de práctica (Figura 1). 
Figura 1

Modelo de Comunidades de práctica CoP

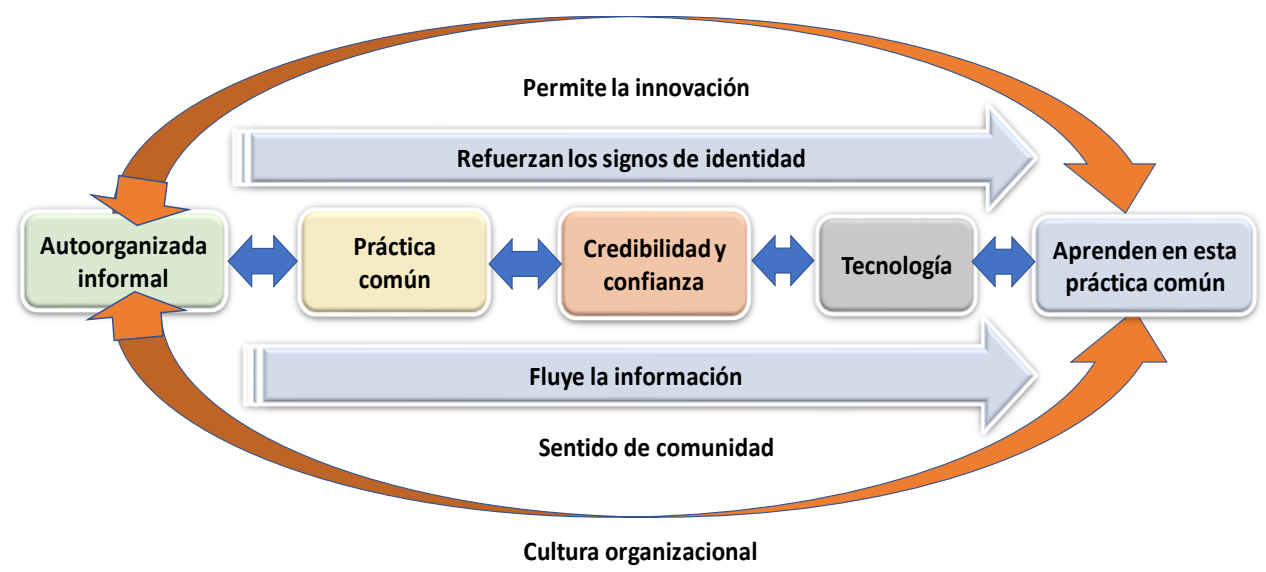

Fuente: Elaboración propia (2018) con base en los aportes de Wenger (1998); Lesser y Prusak (1999); Jubert (1999); Choo (1999); García (2000); Wenger y Snyder (2000); Pickett (2002); Wenger, McDermott y Snyder (2002); Medellín (2003); Chen, Hsin y Seng (2007); Rodríguez (2008).

\section{Cómo poner en marcha comunidades de práctica.}

Con base en los aportes de los autores Brown y Duguid (2000), y la Tabla 5 muestra algunas orientaciones para el diseño y la implementación de las comunidades de práctica.

\section{Tabla 5}

\section{Cómo poner en marcha CoP's}

\begin{tabular}{|c|l|c|}
\hline Orientaciones & \multicolumn{1}{c}{ Descripción } \\
\hline $\begin{array}{c}\text { Sembrar } \\
\text { Establecer propósitos comunes }\end{array}$ & $\begin{array}{l}\text { Nace de forma espontáneamente } \\
\text { Liderazgo espontáneo }\end{array}$ & $\begin{array}{l}\text { Lo debe asumir un miembro que sea aceptado por todos } \\
\text { Grados de aporte }\end{array}$ \\
Confianza & $\begin{array}{l}\text { Sequeden clasificar en: Entusiastas, dinámicos, y aledaños o red de práctica } \\
\text { Re trabajar en grupos pequeños }\end{array}$ \\
\hline Entramado de relaciones & $\begin{array}{l}\text { Se debe lograr una gran participación, con números eventos sincrónicos y } \\
\text { asincrónicos en lo virtual y presenciales } \\
\text { Diseño del lugar de trabajo on-line }\end{array}$ & $\begin{array}{l}\text { Establecer la mejor ergonomía para el puesto el trabajo apoyado con } \\
\text { tecnología }\end{array}$ \\
\hline
\end{tabular}

Fuente: Elaboración propia (2018).

La tabla anterior se complementa con la siguiente explicación:

Sembrar: para poner en marcha las CoP, lo más importante es "sembrar una semilla", es decir, incentivar desde la alta dirección el apoyo para el nacimiento de forma espontánea y el desarrollo de estas. 
Propósitos comunes: con base en la siembra realizada, la CoP inicial debe focalizar sus áreas de interés, consultando a sus miembros: ¿Qué temas son importantes para todos?; ¿De cuáles se establecen conversaciones en los tiempos de descanso?; para así establecer propósitos comunes.

Líder espontáneo: es necesario que la CoP cuente con un líder que impulse y anime la participación de todos sus miembros, compartiendo sus conocimientos, sus ideas nuevas y estableciendo buenas relaciones entre ellos mismos.

Grado de aporte: en cualquier CoP se presentan diferentes tipos de participación: el 15\% como entusiastas, $20 \%$ como dinámicos y finalmente los aledaños que participan eventualmente y que requieren motivación para conformar la comunidad o la red de práctica.

Confianza: esta representa un papel fundamental porque al iniciar la comunidad no se logra más de un $10 \%$ de confianza y ésta se construye día a día, por tanto, se recomienda conformar comunidades de práctica entre 3 a 7 personas, buscando poner en práctica el método Aprendizaje-acción.

Entramado de relaciones: es necesario llevar a cabo múltiples acciones, virtuales y presenciales, que fomenten el intercambio de buenas prácticas entre los miembros de la comunidad, con las virtuales se logran conversaciones dinámicas y permanentes, mientras que con las presenciales se busca generar un equilibrio entre lo que se comparte, la confianza que se ha generado y lo que se aplica.

Diseño del lugar de trabajo on-line: se debe lograr una relación entre la tecnología y el lugar de trabajo, esto requiere la aplicación de los principios de la ergonomía para tal fin.

Como complemento en la Tabla 6, según los autores Wenger y Wenger (2015) se proponen tres condiciones necesarias para conformar una CoP.

\section{Tabla 6}

Condiciones para definir una COP

$\begin{array}{ccl}\text { Condiciones } & \text { Concepto } \\ 1 \text { El dominio } & \text { Se determina con base en el dominio de interés que tienen sus miembros, } \\ \text { acompañada de las destrezas y habilidades que los caracteriza. } & \text { El objetivo es aprender entre todos y de todos, en un lugar físico o virtual que les } \\ 2 & \text { La comunidad } & \text { La condición sine quanon es que los participantes deben tener una práctica } \\ 3 & \text { compartida, con competencias, experiencia y solución de problemas repetidos. }\end{array}$

Fuente: Elaboración propia (2018). 
De esta manera, se destaca la importancia de diferenciar lo que se considera una comunidad de aprendizaje y otro tipo de formas de trabajo en grupo que tienen similitudes con éstas que no pueden denominarse de esta forma. En este orden de ideas, se ubica el aporte de Gairín, Rodríguez y Barrera (2016) proponiendo diferencias entre una CoP y otros tipos de forma de trabajo, las cuales se relacionan a continuación.

\section{Tabla 7.}

\section{Características de las CoP's}

\begin{tabular}{|c|c|c|c|c|}
\hline $\begin{array}{l}\text { Características } \\
\text { de la } \\
\text { organización }\end{array}$ & Propósito & $\begin{array}{l}\text { Forma de } \\
\text { vinculación }\end{array}$ & Afinidad & $\begin{array}{c}\text { Tiempo de } \\
\text { funcionamiento }\end{array}$ \\
\hline CoP & $\begin{array}{lr}\text { La construcción, } & \text { el } \\
\text { intercambio } & \text { de } \\
\text { conocimientos y } & \text { el } \\
\text { desarrollo } & \text { de } \\
\text { competencias de los que } \\
\text { la conforman }\end{array}$ & $\begin{array}{l}\text { Decisión autónoma } \\
\text { de participar }\end{array}$ & $\begin{array}{lr}\text {-Ímpetu } & \\
\text {-Responsabilidad } & \\
\text {-Afinidad en la } & \\
\text { experticia } & \text { de } \\
\text { conocimientos que } & \\
\text { tiene la comunidad } & \end{array}$ & $\begin{array}{l}\text { Cuando el motivo que } \\
\text { los motivó a iniciar } \\
\text { desaparece }\end{array}$ \\
\hline $\begin{array}{l}\text { Colectivos de } \\
\text { interés }\end{array}$ & $\begin{array}{l}\text { Cooperar, aprender, } \\
\text { generar conocimiento }\end{array}$ & $\begin{array}{l}\text { Decisión voluntaria } \\
\text { y abierta. }\end{array}$ & $\begin{array}{l}\text { Compromiso y afinidad } \\
\text { de intereses. }\end{array}$ & $\begin{array}{l}\text { Cuando terminen los } \\
\text { intereses comunes. }\end{array}$ \\
\hline $\begin{array}{l}\text { Equipos de } \\
\text { trabajo formal }\end{array}$ & $\begin{array}{l}\text { Diseñar un servicio o un } \\
\text { producto }\end{array}$ & $\begin{array}{l}\text { La decisión de la } \\
\text { organización }\end{array}$ & $\begin{array}{l}\text { Requisitos del trabajo } \\
\text { y objetivos que los } \\
\text { une. }\end{array}$ & $\begin{array}{l}\text { Hasta la próxima } \\
\text { convocatoria. }\end{array}$ \\
\hline $\begin{array}{l}\text { Equipo de } \\
\text { proyectos }\end{array}$ & $\begin{array}{l}\text { Elaboración de un } \\
\text { proyecto }\end{array}$ & $\begin{array}{l}\text { Decisión de los } \\
\text { directivos de la } \\
\text { organización. }\end{array}$ & $\begin{array}{l}\text { Indicaciones y } \\
\text { objetivos para la } \\
\text { gestión de proyectos. }\end{array}$ & $\begin{array}{l}\text { Cuando se termine el } \\
\text { proyecto. }\end{array}$ \\
\hline $\begin{array}{c}\text { Red } \\
\text { organizacional }\end{array}$ & $\begin{array}{l}\text { Desarrollar acciones sobre } \\
\text { un conocimiento } \\
\text { específico. }\end{array}$ & $\begin{array}{l}\text { Conformación } \\
\text { desde la dirección } \\
\text { de la organización. }\end{array}$ & $\begin{array}{l}\text { Exigencia del trabajo y } \\
\text { tener metas en común. }\end{array}$ & $\begin{array}{l}\text { Hasta que se } \\
\text { terminen las acciones } \\
\text { que los convocaron. }\end{array}$ \\
\hline $\begin{array}{l}\text { Red desde la } \\
\text { organización } \\
\text { informal }\end{array}$ & $\begin{array}{l}\text { Compartir conocimientos } \\
\text { sobre temas en común. }\end{array}$ & $\begin{array}{l}\text { Participación } \\
\text { voluntaria } \\
\text { (amigos, } \\
\text { compañeros de } \\
\text { trabajo entre } \\
\text { otros) }\end{array}$ & $\begin{array}{l}\text { Propósitos individuales } \\
\text { mútuos. }\end{array}$ & $\begin{array}{l}\text { Cuando se termine el } \\
\text { propósito común. }\end{array}$ \\
\hline
\end{tabular}

Fuente: Elaboración propia (2018).

Igualmente es necesario considerar que siguiendo a Wenger y Wenger (2015), las comunidades de práctica producen interés para su conformacion en las organizaciones, debido a que generan el desarrollo de competencias estratégicas como: 
> Capacidad para asumir la gestión del conocimiento como una obligación de la comunidad de práctica logrando desarrollar los requerimientos para su puesta en marcha

$>$ Capacidad para construir un vínculo entre aprendizaje y desempeño organizacional

> Capacidad para afrontar los aspectos operativos y cambiantes del compartir buenas prácticas

$>$ Competencias para desarrollar sus actividades sin límites geográficos u organizacionales

A demás, es importante determinar las ventajes que generan las comunidades de práctica en las organizaciones, por lo tanto en la Tabla 8 y con base en los autores Gairín, Rodríguez y Barrera (2016) se distinguen entre las que surgen a corto y largo plazo.

\section{Tabla 8}

Ventajas de las Comundades de práctica

\begin{tabular}{|c|c|c|}
\hline Participantes & A corto plazo & A largo plazo \\
\hline \multirow{5}{*}{ Miembros } & Aportan al logro de los objetivos & $\begin{array}{l}\text { Consolidan entramados y facilitan el acceso a } \\
\text { buenas prácticas }\end{array}$ \\
\hline & $\begin{array}{l}\text { Permiten el desarrollo de competencias y } \\
\text { conocimientos expertos. }\end{array}$ & Impulsan el desarrollo de sus integrantes \\
\hline & Construyen confianza & $\begin{array}{l}\text { Fomentan el aprendizaje a nivel individual y de } \\
\text { equipo. }\end{array}$ \\
\hline & $\begin{array}{l}\text { Impulsan el esparcimiento entre sus } \\
\text { miembros }\end{array}$ & Consolidan la reputación de la organización \\
\hline & $\begin{array}{l}\text { Aportan un trabajo que le sirve a sus } \\
\text { integrantes }\end{array}$ & $\begin{array}{l}\text { Se fortalecen el reconocerse a sí mismo como } \\
\text { integrante de la comunidad u la organización }\end{array}$ \\
\hline \multirow{4}{*}{ Organización } & Aporta a la solución de problemas & Aporta en la formulación de estrategias nuevas \\
\hline & $\begin{array}{l}\text { Permite el ahorro de tiempo } \\
\text { Genera simbiosis entre áreas de la } \\
\text { organización }\end{array}$ & $\begin{array}{l}\text { Mantiene actualizada la organización } \\
\text { Son una estrategia de retención del talento } \\
\text { humano }\end{array}$ \\
\hline & Fomenta la reutilización de recursos & Desarrolla capacidades estratégicas \\
\hline & $\begin{array}{l}\text { Es un semillero de innovación (creación, } \\
\text { desarrollo y aplicación de conocimiento) }\end{array}$ & Perfecciona los procesos de toma de decisiones \\
\hline
\end{tabular}

De la tabla anterior, se puede inferir que para los miembros de las comunidades como para las organizaciones es muy conveniente su puesta en marcha, ya que genera un gana-gana, que se logra debido a la simbiosis que se da entre éstas y el impacto de las mismas. Para esto se deben considerar los siguientes aspectos que se relacionan en la Tabla 9. 
Tabla 9.

Cómo lograr integrar las CoPa la organización

\section{Características Condiciones}

Voluntad y tiempo para forma parte de la comunidad de práctica

1 Administración Conformadas por colaboradores con reconocimiento y respeto en la organización

Que profesen y practiquen valores esenciales

La alta dirección debe estar involucrad en su puesta en marcha y desarrollo.

$2 \quad$ Exigencias

Se debe conforma la comunidad por personas con interés y compromiso.

Los participantes deben participar en todas las actividades que se programen bien sean presenciales o virtuales para compartir su con conocimiento

Que se tenga el soporte tecnológico para compartir el conocimiento

3 Tecnología Que todos miembros de la comunidad puedan utilizar la tecnología para compartir sus aportes

$4 \quad$ Miembros de la

Tener la voluntad para compartir su conocimiento

comunidad

Compartir el conocimiento sobre las temáticas importantes para la comunidad.

Fuente: elaboración propia (2018) con base en McDermont y O'dell, (2001)

Para lograr un impacto positivo en las personas que conforman las comunidades de práctica y la organización, es necesario que se trabajen desde su origen y las diferentes etapas del ciclo de vida de cada una de ellas, como lo propone Barrera (2015).

\section{Ciclo de vida de las comunidades de práctica.}

Las etapas por las que se desenvuelven las comunidades de práctica han sido objeto de investigación desde los inicios del siglo XXI y los aportes más significativos coinciden en que desde su germinación las comunidades pasan por diferentes ciclos de vida: potencial, en proceso de integración, activas y activas típicas; dispersas y memorables. En la Tabla 10 y en la Figura 2 se muestran las etapas del desarrollo que tienen las comunidades de prácticas según los autores (Gongla y Rissuto, 2001; Wenger et ál., 2002; Pérez y Martínez, (2008); Armengol, Navarro y Carnicero, 2015).

\section{Tabla 10.}

Etapas de desarrollo de las COP

\begin{tabular}{|c|c|c|}
\hline Fases & Descripción & Autores \\
\hline & Es la fase previa de formación de la & Gongola y Rizzuto (2001) \\
\hline Fase potencial & comunidad que está determinada por los & Wenger et ál. (2002) \\
\hline & $\begin{array}{l}\text { intereses, problemas o actividades a realizar } \\
\text { En esta fase se empieza a definir en la }\end{array}$ & Pérez y Martínez (2008) \\
\hline Fase en proceso de & comunidad sus principios operativos, la & Gongola y Rizzuto (2001) \\
\hline integración & $\begin{array}{l}\text { definición de objetivos y la forma de } \\
\text { vinculación. }\end{array}$ & Wenger et ál. (2002) \\
\hline Fase Activa & Se exploran las formas de & Armengol, Navarro y Carnicero (2015) \\
\hline
\end{tabular}




\section{Manuel Garzón Castrillón}

responsabilidades y roles, inicio de la

Pérez y Martínez (2008)

transferencia de conocimientos

Los miembros ya no se vinculan de forma

Fase dispersa intensiva, pero hay comunicación permanente

Gongla y Rizzuto, (2001) entre ello.

Wenger et ál. (2002)

La comunidad deja de ser central pero sus

Fase memorable integrantes la recuerdan como algo memorable en su vida laboral y los resultados siguen siendo útiles.

Fuente: elaboración propia (2018).

Armengol, Navarro y Carnicero (2015)

Wenger et ál. (2002)

Figura 2.

Etapas de desarrollo de las CoP

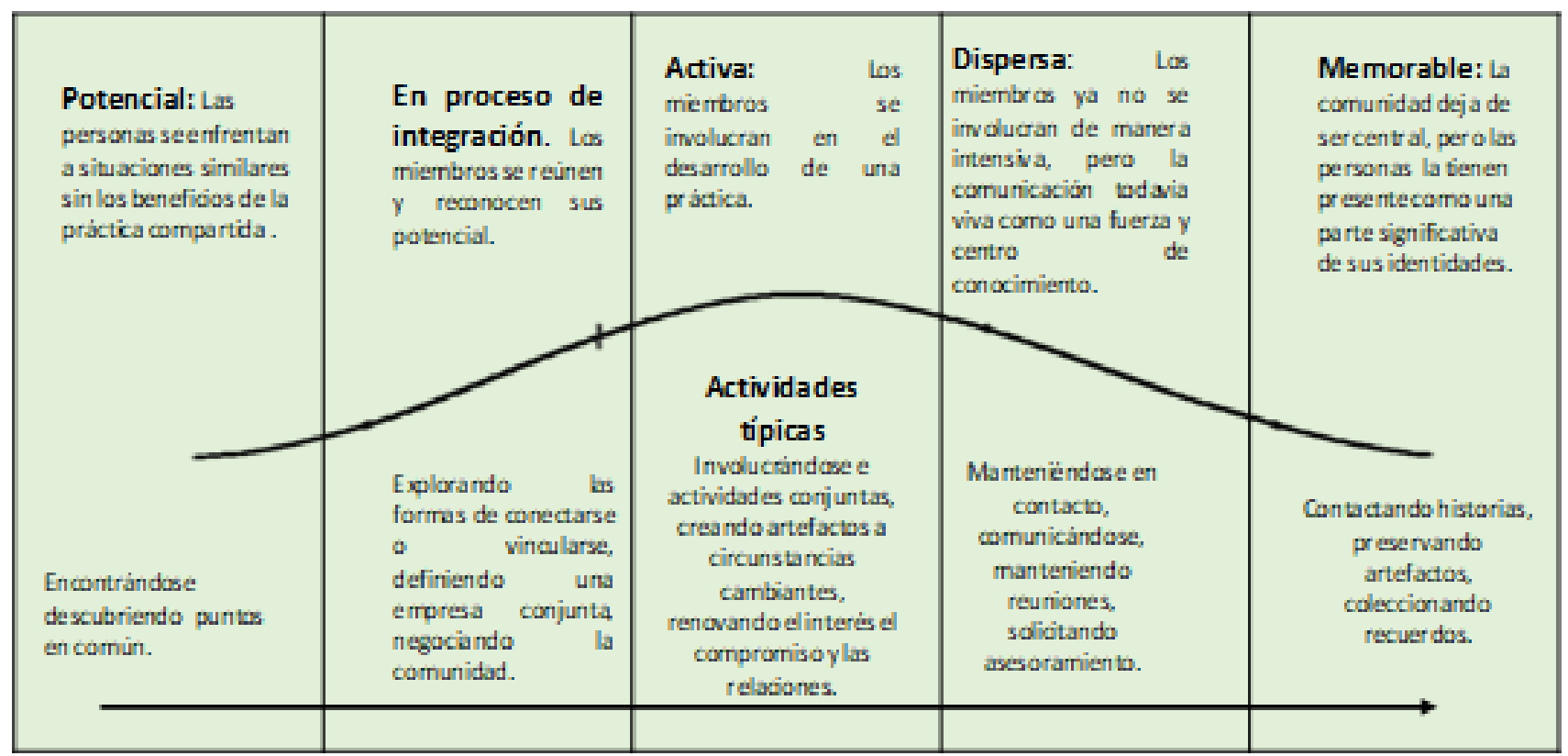

Fuente: elaboración propia (2018).

\section{Componentes de una CoP.}

En el proceso de revisión se encontró que Wenger (1998) fue quien propuso las dimensiones que conforman una comunidad de práctica (Figura 3). Sin embargo, antes de describir las dimensiones es relevante tener en cuenta la definición que este autor expuso con relación a una CoP a lo largo de tres dimensiones. En este sentido, para este autor, una CoP se define como:

Empresa conjunta comprendida y continuamente renegociada por sus miembros, el compromiso mutuo que une a sus miembros juntos en una entidad social y el repertorio compartido de recursos comunes (rutinas, sensibilidades, artefactos, vocabulario, estilos...) que los miembros han desarrollado a lo largo del tiempo. (p. 86) 
Figura 3.

Componentes de una COP

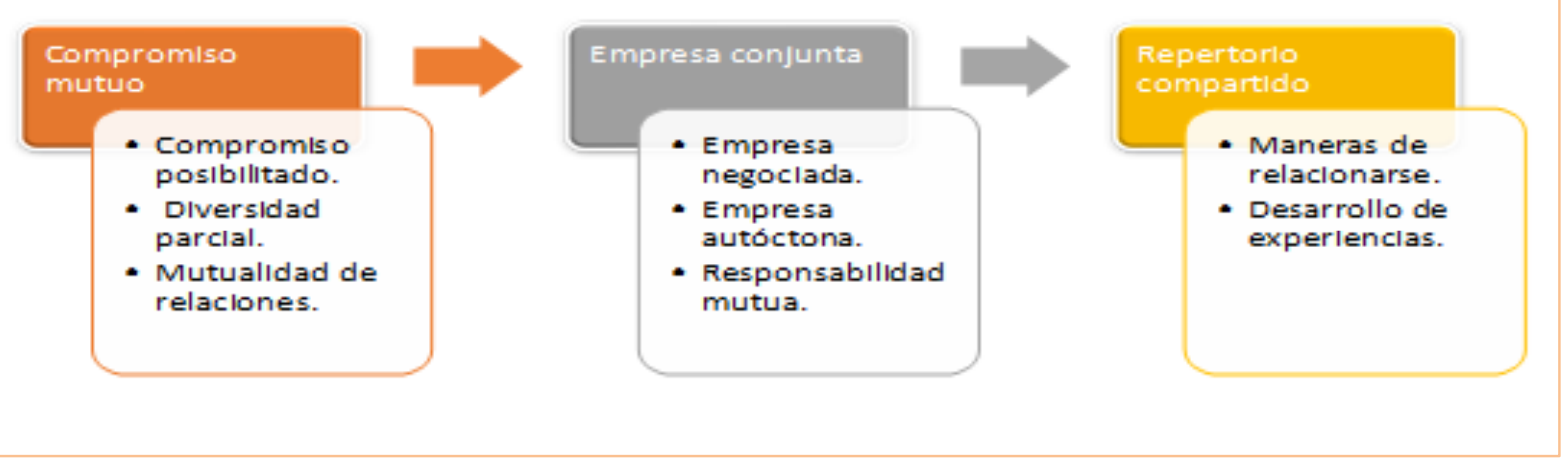

Fuente: elaboración propia (2018).

Las dimensiones que componen una CoP se describen a continuación:

\section{Compromiso.}

Se refiere al comportamiento orientado a compartir el conocimiento que tienen los miembros de la CoP, logrando imprimirle valor, como lo plantea Wenger (1998), esto permite coherencia con el concepto de comunidad, y con las actividades concretas que realizan, los objetivos que se establecen en consenso y su permanente interrelación. Esto se logra con base en el sentido de pertenencia a la comunidad y el intercambio de conocimientos permanente bien sea presencial como virtual, sincrónico o asincrónico.

\section{Diversidad.}

Los miembros de las CoP's son únicos con su conocimiento e identificación con los objetivos de la comunidad, relacionándose con sus colegas con base en las actividades que se realizan y sus aportes diversos resultados para Wenger (1998) de su experiencia y conocimientos que pueden presentar parcialidad concebida esta característica como una capacidad o como una limitante.

\section{Relaciones mutuas.}

Las relaciones son la base de la CoP, que surgen por los acuerdos logrados al entrar a formar parte de la comunidad, que no está libre de conflictos, pues éstos se presentan en toda organización humana, que reflejaran la colaboración en la práctica de la CoP, en las cuales se pueden manifestar en discusiones acaloradas, desacuerdos que para Wenger (1998) son la demostración de un mayor compromiso que si estos comportamientos fueran pasivos o conformistas. 


\section{Sociedad conjunta.}

Los objetivos que persiguen los miembros de la CoP deben ser comunes, pues esto muestra la voluntad de conformar una sociedad conjunta, en la cual se pueden presentar.

\section{Sociedad negociada.}

Requiere de discusión y convenio de compromiso mutuo, resultado de la negociación realizada para establecer los objetivos que se perseguirán en conjunto.

\section{Sociedad autóctona.}

Dentro de las CoP's las actividades son autóctonas resultados de los aportes individuales de cada uno de los integrantes que la conforman y se reflejan en las interacciones diarias que se desarrollan al interior de las comunidades.

\section{Mutua responsabilidad.}

Esta es una condición sine qua non, en el que la responsabilidad de las acciones realizadas en la CoP es de todos los que la conforman, y así se debe acordar desde el inicio.

\section{Prácticas compartidas.}

En el desarrollo de las acciones propias de la CoP se desarrollan prácticas que se manifiestan en expresiones con significado compartido, procesos, formas de interrelacionarse que para Wenger (1998) son producto del funcionamiento de la comunidad, que puede estar apoyado por tecnología (Sanz, 2005) de forma virtual conformándose en comunidades de práctica virtual, con ubicación de sus miembros en husos horarios diferentes (Lesser y Stok, 2001).

\section{Aspectos que contribuyen al éxito de las CoP.}

En los párrafos anteriores se han realizado aproximaciones a las fases por las que atraviesan las comunidades de práctica durante su existencia, por lo tanto, es de vital importancia hacer un acercamiento a los aspectos que contribuyen al éxito de las CoP.

En este aspecto, autores como Gairín, Rodríguez y Barrera (2015) desarrollaron una investigación en la que el principal objetivo fue analizar los factores que condicionan el éxito de las CoP. En la Tabla 11, se muestran que elementos claves para el éxito de la comunidades de práctica, se consolidan en cuatro dimensiones: Organizacionales, Dinámica de la CoP, Personal de los participantes y Resultados que a su vez se dividen en las siguientes categorías: Infraestructura, Cultura organizacional, Rol de los líderes, Motivación, Reconocimientos, Contenido CoP, el Moderador, Reuniones sincrónicas, Objetivos, Motivacionales, De conocimiento, Actitudinales, Aporte a la organización, Clima organizacional y Formación. 


\section{Manuel Garzón Castrillón}

\section{Tabla 11}

\section{Aspectos que contribuyen al éxito de las COP}

\begin{tabular}{|c|c|c|}
\hline Dimensión & Categorías & Aspectos que contribuyen al éxito \\
\hline \multirow{23}{*}{ Organizacionales } & & Recursos técnicos, tecnológicos y materiales necesarios para sus actividades \\
\hline & Infraestructura & Apoyo virtual sincrónico y asincrónico \\
\hline & & Funciones dentro del horario de trabajo \\
\hline & & Voluntad para compartir conocimientos \\
\hline & $\begin{array}{l}\text { Cultura } \\
\text { organizacional }\end{array}$ & Clima organizacional que fomente la gestión del conocimiento. \\
\hline & & Ser miembro de la CoP es parte de su trabajo \\
\hline & \multirow{4}{*}{ Rol de los líderes } & Comprender las solicitudes y peticiones que hagan los miembros. \\
\hline & & Comprometidos con el funcionamiento de la CoP \\
\hline & & Hacer seguimiento a los objetivos de la CoP \\
\hline & & Facilita la incorporación de las buenas prácticas en las actividades laborales \\
\hline & \multirow{3}{*}{ Motivación } & Asignación de tiempo para participar en la CoP \\
\hline & & $\begin{array}{l}\text { Reconocimiento público por los aportes } \\
\text { Reconocimiento público de participación y e interés de seguir vinculado a la } \\
\text { comunidad }\end{array}$ \\
\hline & & Integración de las buenas prácticas al trabajo de la organización. \\
\hline & \multirow{3}{*}{ Reconocimientos } & Propiedad intelectual (moral) de los autores \\
\hline & & Certificación de la participación en la CoP \\
\hline & & Valoración de los aportes realizados \\
\hline & \multirow[t]{4}{*}{ Contenido CoP's } & Atractivo y útil para los usuarios \\
\hline & & Orienta a los miembros hacia los propósitos establecidos \\
\hline & & Organiza y hace seguimiento a los compromisos de cada participante \\
\hline & & Crea clima propicio para construir nuevo conocimiento. \\
\hline & \multirow{5}{*}{ Moderador } & Retroalimentación a los participantes \\
\hline & & Fortalece la interacción de los miembros \\
\hline & & Apoya la solución de problemas técnicos \\
\hline \multirow[t]{8}{*}{ Dinámica de la CoP } & & Divulga los resultados de la CoP \\
\hline & & Logra mesura entre las actividades del día a día y las de la CoP \\
\hline & $\begin{array}{l}\text { Reuniones } \\
\text { sincrónicas }\end{array}$ & Busca la relación directa de los participantes superando la logrado virtualmente \\
\hline & Objetivos & Claros y logrables, acordados desde el inicio \\
\hline & & Resultado de la participación de los miembros \\
\hline & & Creencia que los aportes a la CoP son importantes para su trabajo \\
\hline & Motivacionales & Participación en las actividades de la CoP \\
\hline & & Abierto al aprendizaje permanente para mejorar su trabajo \\
\hline \multirow{9}{*}{$\begin{array}{l}\text { Personal de los } \\
\text { participantes }\end{array}$} & \multirow{3}{*}{ De conocimiento } & Conoce las herramientas utilizadas en la CoP \\
\hline & & Tiene conocimientos importantes para aportar a la CoP \\
\hline & & Compromiso con las actividades asignadas \\
\hline & \multirow[t]{3}{*}{ Actitudinales } & Asume responsabilidades que puede cumplir \\
\hline & & Presenta con argumentos en la CoP \\
\hline & & Los resultados aportan a la organización \\
\hline & \multirow{3}{*}{$\begin{array}{l}\text { Aportan a la } \\
\text { organización }\end{array}$} & Permiten mejoramiento en las labores diarias \\
\hline & & Son referentes para la organización \\
\hline & & Difusión de los resultados \\
\hline \multirow[t]{2}{*}{ Resultados } & $\begin{array}{c}\text { Clima } \\
\text { organizacional }\end{array}$ & $\begin{array}{l}\text { Las actividades realizadas mejoran el clima laboran. } \\
\text { Consolidan las relaciones entre los miembros de la organización } \\
\text { Inspiran la consolidación de conciencia colectiva }\end{array}$ \\
\hline & Formación & $\begin{array}{l}\text { Favorecen el aprendizaje de los miembros de la CoP y de la organización } \\
\text { Apoyan a los nuevos colaboradores con sus resultados }\end{array}$ \\
\hline
\end{tabular}


Funciones de moderador de la Comunidad de práctica.

El rol del moderador es muy importante para el funcionamiento de la CoP, para Vásquez (2001) es el dinamizador de la comunidad que fortaleciendo la actividad y compartiendo el conocimiento en forma tácita y explícita, además de infundir respeto entre la comunidad. Este líder desde el enfoque de Wenger et ál., (2002) debe lograr sinergia de las actividades a ejecutar por la CoP. En la Tabla 12 se relacionan las funciones más importantes:

\section{Tabla 12}

Funciones del moderador de la CoP

$\begin{array}{ll}1 & \text { Establecer los asuntos a tratar en la CoP } \\ 2 & \text { Impulsar el desarrollo de los asuntos a realizar } \\ 3 & \text { Optimizar el desarrollo de los miembros } \\ 4 & \text { Permitir la consolidación de redes entre los miembros y la gestión del conocimiento } \\ 5 & \text { Servir de canal de comunicación entre la CoP y la organización } \\ 6 & \text { Impulsar la construcción del conocimiento } \\ 7 & \text { Conseguir los elementos necesarios para las actividades a realizar } \\ 8 & \text { Buscar el desempeño adecuado de la CoP evaluando su funcionamiento }\end{array}$

Fuente: Elaboración propia (2018).

De la misma forma es necesario que el moderador sea un experto del tema o los temas a tratar en la CoP, con la autonomía necesaria para lograr los objetivos establecidos por sus miembros (Sanz, 2005).

\section{Método}

Para la realización de esta revisión, la metodología utilizada fue de tipo cualitativo, corte descriptivo. Para el desarrollo del estudio se realizó tres etapas: 1) búsqueda exhaustiva de la bibliografía; 2) organización de los datos; y 3) análisis de contenido.

Para este proceso, se estudiaron (58) cincuenta y ocho documentos en fuentes secundarias los cuales fueron consultados en de base de datos Scopus, WoS, y Scielo.

\section{Evaluación critica}

Dentro de la revisión de la literatura varios autores han expresado sus preocupaciones sobre la operacionalización de las Comunidades de Prácticas y enfatizan que las perspectivas instrumentales de éstas a menudo conducen a perder de vista su énfasis original en la identidad y el contexto social, así como también un desvío de la filosófica del concepto hacia la ontología realista. De esta manera, teniendo 


\section{Manuel Garzón Castrillón}

en cuenta esto, la noción de las CoP's se considera fundamental para el aprendizaje organizacional y a diferencia de los equipos, éstas no desempeñan un papel formal en las organizaciones como tampoco están libres de luchas de poder, lo que parece importante garantizar que una Comunidad de Práctica dentro de una organización si puede funcionar, por tanto es necesario que las CoP's sean legitimadas por los directivos en las organizaciones con el fin de cultivar entornos de trabajo donde las personas estén comprometidas, para que puedan asumir la responsabilidad de su aprendizaje y su práctica.

Asimismo, el concepto de CoP no debe considerarse obsoleto en el contexto de la literatura actual, por el contrario, aún debe desarrollarse más y desarrollarse, en razón de aquellas organizaciones que sobresalen y aprovechan al máximo el conocimiento de sus empleados ya que serán aquellas lo suficientemente audaces como para alentar a sus colaboradores a comprometerse, asumir riesgos, aceptar las enseñanzas de los demás y crecer efectivamente como trabajadores competentes.

\section{Contribución del autor}

En primer lugar, es importante destacar que este artículo de revisión contribuye al conocimiento sobre las comunidades de práctica porque consolida el conocimiento existente sobre el tema, empezando por abordar el concepto de comunidad, para pasar al de comunidades de práctica y como resultado del análisis de los diferentes enunciados, se propone una definición consolidada.

También se realiza un recorrido por los antecedentes y desarrollo de las comunidades de práctica, con base en lo cual se hacen aproximaciones que permitan la puesta en marcha de las CoP's, estableciendo sugerencias para el diseño y el ciclo de vida que puede recorrer la comunidad, abordando los elementos que pueden facilitar la obtención de éxitos en su puesta en marcha.

Lo anterior, se establece teniendo en cuenta que el trabajo apoyado en las CoP's, permite a las organizaciones impulsar el trabajo colaborativo orientado al logro de propósitos comunes. De la misma forma las CoP's permiten el uso del conocimiento existente de forma tácita y explícita, que permiten a la organización dar respuesta a los cambios dinámicos del entorno.

\section{Conclusiones}

En este artículo de revisión abordó un tema vital para el funcionamiento de las organizaciones, teniendo en cuenta que el conocimiento es dinámico, una alternativa para compartirlo y construirlo, son las CoP's, que puestas en marcha permitirán el logro de mejores resultados empresariales. 
Desde el paradigma del Conocimiento-Capacidades-Performance (KCP), las comunidades de práctica, idealmente puestas en marcha desde la organización informal, son una herramienta fundamental para el aprendizaje organizacional efectivo. Asimismo, es importante tener en cuenta que para la puesta en marcha de las CoP's, las fases del ciclo de vida que se identifican son Fase potencial; Fase en proceso de integración; Fase Activa; Fase dispersa; Fase memorable, las cuales son fases claves para lograr los resultados esperados.

De esta manera, se puede concluir que para las organizaciones que decidan poner en marcha de comunidades de práctica, es fundamental tener en cuenta las cuatro dimensiones (Organizacionales, Dinámica de la CoP, Personal de los participantes y Resultados) que permitirán una puesta en marcha exitosa.

Finalmente, se destaca la importancia del rol del moderador, cuya función es primordial para el logro del éxito de una CoP, ya que es quien debe lograr la sinergia, la relación permanente, la transferencia del conocimiento tácito y explícito de las buenas prácticas de los miembros con toda la organización.

\section{Referencias}

Armengol, C., Navarro, M. y Carnicero, P. (2015). La creación de comunidades de práctica profesional. En Gairín, J. (Coord.). Comunidades de Práctica Profesional. Creación, desarrollo y evaluación (pp. 33-50). Madrid: Wolters Kluwer.

Barrera-Corominas, A. (2015). La transferencia de los aprendizajes adquiridos en comunidades de prácticas en la administración pública (Tesis doctoral). Universitat Autónoma de Barcelona).

Brown. J. \& Duguid, P. (1991). Organizational learning and communities of practice. Organization Science, 2(1), 50-57. https://www.jstor.org/stable/2634938?seq=1

Chen-Ya. W., Hsin-Yi. \& Seng-Cho, C. (2007). Using peer-to-peer technology for knowledge sharing in communities of practices. Decision Support Systems, 45(3), 528-540. https://doi.org/10.1016/j.dss.2007.06.012

Choo, C. (1999). La Organización inteligente. [Traducido al español de Daniel Rey]. México: Editorial Oxford Press.

Coll, C., Bustos, A. y Engel, A. (2007). Configuración y evolución de la comunidad virtual MIPE/DIPE: Retos y dificultades. Revista Electrónica Teoría de la Educación. Revista Interuniversitaria, 8(3), 87104. http://campus.usal.es/ teoriaeducacion/DEFAULT.html 
Corominas, J. (1987). Breve diccionario etimológico de la Lengua Castellana. Madrid: Editorial Gredos.

Gairín, J., Rodríguez-Gómez D. \& Barrera-Corominas, A. (2016). Hints for Rethinking Communities of Practice in Public Administration: An Analysis from Real Practice. In Buckley, S., Majewski, G. \& Giannakopoulos, A. (Eds.). Organizational Knowledge Facilitation through Communities of Practice in Emerging Markets. Hershey: IGI Global.

Gallego, D. y Valdivia, J. (2013). Las comunidades de prácticas virtuales: Un espacio de participación para la mejora de las prácticas educativas del profesorado. Madrid: Editorial DYKINSON S.L.

Garber, D. (2004). Growing virtual communities. International Review of Research in Open and Distance Learning, 5(2), 1-7. https://doi.org/10.19173/irrodl.v5i2.177

García-Blanco, M. (2000). El aprendizaje del estudiante para profesor de matemáticas desde la naturaleza situada de la cognición: Implicaciones para la formación inicial de maestros. En Zurbano, C. (Ed.). Propuestas metodológicas y de evaluación en la Formación Inicial de los Profesores el Área de Didáctica de las Matemáticas (pp.55-79). Oviedo: Universidad de Oviedo.

Gongla, P. \& Rizzuto, C. R. (2001). Evolving communities of practice: IBM Global Services Experience. IBM Systems Journal, 4O(4), 842-862. https://ieeexplore.ieee.org/document/5386943

Hamman, R. (2001). Computer Networks Linking Networks Communities. In Werry, C. y Mowbray, M. (Eds.), Online Communities (pp. 72-92). México: Prentice Hall.

Hilley, G. (1955). Definitions of Community: Areas of Agreement. Rural Sociology, 20(2), 118-125.

Jubert, A. (1999). Developing an infrastructure for communities of practice. In Mckenna, B. (Ed.). Proceedings of the 19th International Online Meeting. Oxford: Learned Information.

Lesser, E. \& Prusak, L. (1999). Communities of practice, social capital and organizational knowledge. Cambridge: IBM Institute for Knowledge Management.

Lesser, E. L. \& Storck, J. (2001). Communities of practice and organizational performance. IBM Systems Journal, 40(4), 831-841. https://doi.org/10.1147/sj.404.0831

McDermott, R. \& O'Dell, C. (2001). Overcoming cultural barriers to sharing knowledge. Journal of Knowledge Management, 5(1), 76-85. https://doi.org/10.1108/13673270110384428

McMillan, D. W. \& Chavis, D. M. (1986). Sense of Community: A definition and Theory. Am. Journal of Community Psychology, 14(1), 6-23. https://doi.org/10.1002/1520-6629(198601)14:1\%3C6::AIDJCOP2290140103\%3E3.0.CO;2-I 
Medellín, E. (2003). ¿Qué es y para qué sirve la administración del conocimiento? La administración del conocimiento en centros públicos de investigación y desarrollo: el caso Centro de Investigación, en Química Aplicada [tesis de pregrado] UNAM, Ciudad de México, México.

Pérez-Montro, M. y Martínez, J. (Octubre, 2008). Success factors of Communities of in Public Administration: The case of Catalonia's Government. Trabajo presentado en la $5^{\circ}$ Conferencia Internacional sobre Capital Intelectual, Gestión del Conocimiento y Aprendizaje organizacional Nueva York. Instituto de Tecnología, Nueva York, Estados Unidos.

Pickett, J. (2002). the American Heritage Dictionary of the English Language. $4^{\text {th }}$ ed. Boston: Houghton Mifflin.

Pons, J., Gil, M. y Grande, J.M. (1996). Participación y sentimiento de pertenencia en comunidades urbanas: aproximación metodológica a su evaluación. RTS: Revista Treball Social, (141), 32-48. https://dialnet.unirioja.es/servlet/articulo?codigo $=495401$

Poole, M. (January 2002). Developing Online Communities of Practice in Preservice Teacher Education [Conference]. Paper presented in CSCL Conference, Boulder, Colorado. https://dl.acm.org/doi/pdf/10.5555/1658616.1658731

Rodríguez, J. (2008). Comunidades Virtuales de Práctica y de Aprendizaje. Barcelona: Publicaciones i Edicions de la Universitat de Barcelona.

Rodríguez-Gómez, D. Gairín, J. y Armengol, C. (2011). La moderación en el modelo ACCELERA de creación y gestión del conocimiento. En Gairín, J. (Coord.). El trabajo colaborativo en red (pp. 143-160). Barcelona: Davinci Continental.

Sanz, S. (2005). Comunidades de práctica virtuales: acceso y uso de contenidos. Revista de Universidad y Sociedad del Conocimiento, 2(2), 26-35. http://dx.doi.org/10.7238/rusc.v2i2.259

Tönnies, F. (1895). Comunidad y Asociación. Barcelona: Península.

Vásquez, S. (2001). Comunidades de práctica. EDUCAR, 47(1), 51-68. https://doi.org/10.5565/rev/educar.71

Wellman, B. \& Gulia, M., (1999).Virtual Communities as Communities: Net Surfers Don't Ride Alone. In Smith, M. \& Kollock, P. (Eds), Communities in Cyberspace. London: Routledge.

Wenger, E. \& Snyder, W. (2000). Communities of practice: the organizational frontier. Harvard Business Review, 78(1), 139-145. https://hbr.org/2000/01/communities-of-practice-the-organizational-frontier 
Wenger, E. (1998). Communities of Practice: Learning Meaning, and Identity. Cambridge: Cambridge University Press.

Wenger, E. (2001). Comunidades de práctica: aprendizaje, significado e identidad. Barcelona: Paidós.

Wenger, E., McDermott, R. \& Snyder, W. M. (2002). Cultivating Communities of Practice: A guide to Managing Knowledge. Boston: Harvard Business School Press.

Wenger-Trayner, E. y Wenger-Trayne, B. (2015). Comunidades de práctica una breve introducción (Traducción de Govea Aguilar). http://www.pent.org.ar/institucional/publicaciones/comunidadespractica-una-breve-introduccion.

Wilbur, P. (1997). An Archaelogy of Cyberspaces: Virtuality, Community, Identity. En Porter, D. (Comp.). Internet Culture. (pp. 45-55). New York: Routledge. 